typeset using JPSJ.sty $<$ ver.1.0b $>$

\title{
Charge Kondo effect toward a non-Fermi-liquid fixed point in the orbitally degenerate exchange model
}

\author{
Hiroaki Kusunose* and Yoshio Kuramoto \\ Department of physics, Tohoku University, Sendai, 980-8578
}

(Received August 14, 2021)

\begin{abstract}
We show that a Kondo-type model with an orbital degeneracy has a new non-Fermi-liquid fixed point. Near the fixed point the spin degrees of freedom are completely quenched, and the residual charge degrees of freedom lead to the multi-channel Kondo effect. Anomalous behavior appears in electric and thermal properties, but the magnetic susceptibility should show the local Fermi-liquid behavior. The non-Fermi-liquid fixed point becomes unstable against perturbations breaking the particle-hole symmetry. We derive these results using the third-order scaling for a spherically symmetric model with a fictitious spin. In contrast to the Coqblin-Schrieffer model, the present model respects different time-reversal properties of multipole operators.
\end{abstract}

KEYWORDS: orbital degeneracy, time-reversal property, multipole, multi-channel Kondo effect, non-Fermi liquid, $\mathrm{CeB}_{6}, \mathrm{LaMnO}_{3}$

Orbital dynamics has recently attracted much interest because of new experimental progress in heavy-fermion systems such as $\mathrm{CeB}_{6}{ }^{1-3)}$ and transition metal oxides such as $\mathrm{LaMnO}_{3} \cdot{ }^{4,5)}$ In such systems, the localized ground multiplet has an orbital degeneracy $n$ in addition to the Kramers degeneracy. Thus orbital tensors as well as spin ones constitute observables. ${ }^{6-9)}$ The simplest model to describe the combined spin and orbital degrees of freedom is the $\mathrm{SU}(2 n)$ symmetric spin-orbit model. ${ }^{10-14)}$ In this model, however, the different timereversal properties of the spin and orbital tensors are hidden in the excessively high symmetry.

One can naturally ask how the Kondo effect occurs in the presence of the orbital degeneracy, and how the anisotropy in the combined spin-orbit space influences the physical property. In this context we refer to the pioneer work of Hirst who extended the Coqblin-Schrieffer (CS) model for the realistic atomic level structure. ${ }^{15)}$ Pavarini and Andreani recently studied the stability of the local Fermi liquid in the CS model against the symmetry breaking interaction found by Hirst, and concluded that it is stable. ${ }^{16)}$ In order to include the realistic crystalline-electric-field (CEF) effect, the present authors considered more general exchange interaction between $f$ and conduction electrons based on the point-group analysis, and discussed how the time-reversal property of operators is reflected in the Kondo effect. ${ }^{17)}$ In general, operators describing the spin and/or the orbital degrees of freedom are classified according to their definite timereversal properties. The operators which are even under the time reversal are called electric, and those which are odd are called magnetic. The distinction between magnetic and electric characters should be important in interpreting and predicting experimental results including dilution effects.

In this Letter we propose a new mechanism to realize

*E-mail: kusu@cmpt01.phys.tohoku.ac.jp the non-Fermi-liquid fixed point by combination of spin and orbital degrees of freedom. Near the fixed point only the spin degrees of freedom are compensated, and the residual charge degrees of freedom leads to the multichannel Kondo effect. This non-Fermi-liquid fixed point requires the presence of particle-hole symmetry, and the anomalous behavior appears only in electric properties. Namely the magnetic susceptibility behaves as if the system is a local Fermi liquid.

Let us start with our choice of the basis set and explain their time-reversal property. The ground multiplet is written as $|l \sigma\rangle(l=1,2, \cdots n, \sigma=\uparrow, \downarrow)$, where $\sigma$ represents the Kramers pair with arbitrary strong spin-orbit interaction. The time-reversal operation $\theta$ is defined as

$$
\theta|l \sigma\rangle=(-1)^{1 / 2-\sigma}|l-\sigma\rangle .
$$

The conventional Pauli matrices $\sigma^{\alpha}$ acting only on the spin state are odd under time reversal:

$$
\theta \sigma^{\alpha} \theta^{-1}=-\sigma^{\alpha}, \quad(\alpha=x, y, z) .
$$

We define the orbital operators $\tau^{i}$ which act only on the orbital state $l$. Their time-reversal properties are represented as

$$
\theta \tau^{i} \theta^{-1}=\left(\tau^{i}\right)^{*}, \quad\left(i=1,2, \cdots, n^{2}-1\right),
$$

due to the antilinearity of the time-reversal operation. Thus, in the Hermitian representation the pure imaginary orbital operators are odd under time reversal, and have the magnetic character. In the simplest case of $n=2$, the operator $\tau^{y}$ is magnetic and $\tau^{x}$ and $\tau^{z}$ are electric. In the case of $n=3$, three of eight orbital operators are magnetic and five are electric. In the absence of the spin-orbit interaction, magnetic orbital operators describe the orbital moment of electrons.

In order to facilitate the scaling analysis, we map the states onto $|m\rangle$ where $m$ represents the $z$-component of a fictitious spin $j=n-1 / 2$ (half integer). An example 
of mapping for $n=3$ is given by

$$
\begin{aligned}
& |1 \uparrow\rangle \rightarrow|+5 / 2\rangle,|2 \downarrow\rangle \rightarrow|+3 / 2\rangle,|3 \uparrow\rangle \rightarrow|+1 / 2\rangle, \\
& |3 \downarrow\rangle \rightarrow|-1 / 2\rangle,|2 \uparrow\rangle \rightarrow|-3 / 2\rangle,|1 \downarrow\rangle \rightarrow|-5 / 2\rangle .(4)
\end{aligned}
$$

We assume that the Hamiltonian has the spherical symmetry in the new basis set. Then we introduce the spherical tensor operators ${ }^{15,18)}$ which have a simple timereversal property:

$$
\theta T_{q}^{(p)} \theta^{-1}=(-1)^{p}\left[T_{q}^{(p)}\right]^{\dagger},
$$

where each operator represents $2^{p}$ th magnetic (electric) multipole for odd (even) $p$ with the component $q$. This property under time reversal merely reflects the odd character of the first rank operator, the angular momentum.

The set of the spherical tensors with $p=1,2, \cdots, 2 j$ together with the unit matrix completely describes the $2 n \times 2 n$ kinds of transition between local electron states. Then any spherically symmetric interaction among operators between $f$ and conduction electrons is described in terms of the spherical tensors as

$$
H_{\mathrm{int}}=\sum_{p=1}^{2 j} J_{p} \sum_{q=-p}^{p} c^{\dagger} \mathrm{T}_{q}^{(p)} c|f\rangle\left[\mathrm{T}_{q}^{(p)}\right]^{\dagger}\langle f|,
$$

where $J_{p}$ is the coupling constant, $\mathrm{T}_{q}^{(p)}$ is a matrix representation of the tensor operator with dimension $2 n$ and summation over quantum numbers of each state is implied by the matrix multiplication. Here the spherical tensors are orthonormalized as

$$
\operatorname{Tr}\left(\mathrm{T}_{q}^{(p)}\left[\mathrm{T}_{q^{\prime}}^{\left(p^{\prime}\right)}\right]^{\dagger}\right)=\delta_{p p^{\prime}} \delta_{q q^{\prime}}
$$

In general, the angular momentum $j$ of conduction electrons is not necessarily the same as the localized electron. ${ }^{19,20)}$ However, we assume the same $j$ for simplicity. In the case of $J_{p}=J_{\mathrm{CS}}$ for all $p$, one recovers the Coqblin-Schrieffer model with the $\mathrm{SU}(2 n)$ symmetry. It should be emphasized that the spherical symmetry $\mathrm{SU}(2)$ in eq.(6) is adopted just for technical convenience. Obviously the actual system with the $\mathrm{CEF}$ is not invariant under the infinitesimal rotation. The simplified model presented above is sufficient for our purpose of analyzing the consequence of different time-reversal properties between electric and magnetic degrees of freedom. Because the time-reversal property of operators is conserved under the discrete rotation of the point-group, the extension of the model is straightforward.

A general formula is available for constructing the scaling equation in terms of the structure constants of the algebra for the related matrices. ${ }^{17,21)}$ The commutation rule is given by

$$
\left[\mathrm{T}_{\alpha}, \mathrm{T}_{\beta}\right]=\sum_{\gamma} i f_{\alpha \beta \gamma}\left[\mathrm{T}_{\gamma}\right]^{\dagger},
$$

where the Greek $\alpha, \beta$ and $\gamma$ are abbreviation of $(p, q)$, $\left(p^{\prime}, q^{\prime}\right)$ and $\left(p^{\prime \prime}, q^{\prime \prime}\right)$, respectively. We adopt the completely antisymmetric structure constant which is given by

$$
f_{\alpha \beta \gamma}=2(-1)^{2 j-\left(p+p^{\prime}+p^{\prime \prime}+1\right) / 2} S_{p p^{\prime} p^{\prime \prime}}^{(j)}\left(\begin{array}{ccc}
p & p^{\prime} & p^{\prime \prime} \\
q & q^{\prime} & q^{\prime \prime}
\end{array}\right),
$$

where the parenthesis including three columns is the Wigner's $3 j$ symbol. ${ }^{18}$ ) The symmetric function $S$ with respect to $p$ 's is defined as

$S_{p p^{\prime} p^{\prime \prime}}^{(j)}=\sqrt{(2 p+1)\left(2 p^{\prime}+1\right)\left(2 p^{\prime \prime}+1\right)}\left\{\begin{array}{ccc}p & p^{\prime} & p^{\prime \prime} \\ j & j & j\end{array}\right\}$,

with $p+p^{\prime}+p^{\prime \prime}=$ odd. The bracket including three columns represent the $6 j$ symbol. ${ }^{18)}$

Once the commutation rule is given, we can easily obtain the scaling equations up to third order:

$$
\begin{gathered}
\frac{\partial}{\partial \xi} J_{p}=\beta_{p}^{(2)}+\beta_{p}^{(3)} \\
\beta_{p}^{(2)}=-\frac{2}{2 p+1} \sum_{p^{\prime} p^{\prime \prime}} J_{p^{\prime}} J_{p^{\prime \prime}}\left(S_{p p^{\prime} p^{\prime \prime}}^{(j)}\right)^{2}, \\
\beta_{p}^{(3)}=\frac{2 J_{p}}{2 p+1} \sum_{p^{\prime}} J_{p^{\prime}}^{2} \sum_{p^{\prime \prime}}\left(S_{p p^{\prime} p^{\prime \prime}}^{(j)}\right)^{2},
\end{gathered}
$$

where the coupling constants are measured in units of the bare cut-off energy $D_{0}$, and $\xi=\ln \left(D / D_{0}\right)$.

Let us simplify the analysis by putting $J_{p}=J_{m}$ for odd $p$ and $J_{p}=J_{e}$ for even $p$. Namely we pay attention only to the difference in time-reversal property of operators. Then eqs. (11)-(13) are reduced to

$$
\begin{aligned}
& \frac{\partial}{\partial \xi} J_{m}=-2\left(1-J_{m}\right)\left(A_{m}^{(j)} J_{m}^{2}+A_{e}^{(j)} J_{e}^{2}\right), \\
& \frac{\partial}{\partial \xi} J_{e}=2 A_{c}^{(j)} J_{e}\left[\left(J_{m}^{2}+J_{e}^{2}\right)-2 J_{m}\right],
\end{aligned}
$$

where $A$ 's are defined as

$$
\begin{aligned}
& A_{m}^{(j)}=\frac{1}{2 p+1} \sum_{p^{\prime} p^{\prime \prime}}^{\text {odd }}\left(S_{p p^{\prime} p^{\prime \prime}}^{(j)}\right)^{2}, \quad(p=\text { odd }), \\
& A_{e}^{(j)}=\frac{1}{2 p+1} \sum_{p^{\prime} p^{\prime \prime}}^{\text {even }}\left(S_{p p^{\prime} p^{\prime \prime}}^{(j)}\right)^{2}, \quad(p=\text { odd }), \\
& A_{c}^{(j)}=\frac{1}{2 p+1} \sum_{p^{\prime}}^{\text {odd even }} \sum_{p^{\prime \prime}}\left(S_{p p^{\prime} p^{\prime \prime}}^{(j)}\right)^{2}, \quad(p=\text { even }) .
\end{aligned}
$$

The summation over $p^{\prime}$ and $p^{\prime \prime}$ can be performed explicitly and $A$ 's are independent of $p$. For half-integer (integer) $j$, we obtain

$$
\begin{aligned}
& A_{m}^{(j)}=\frac{1}{4}(2 j+3), \quad\left(\frac{1}{4}(2 j-1)\right), \\
& A_{e}^{(j)}=\frac{1}{4}(2 j-1), \quad\left(\frac{1}{4}(2 j+3)\right), \\
& A_{c}^{(j)}= \begin{cases}0 & (j=1 / 2), \\
\frac{1}{4}(2 j+1) & (\text { otherwise }) .\end{cases}
\end{aligned}
$$

One can confirm that the scaling equation for the $\mathrm{SU}(2 n)$ CS model is reproduced with $J_{m}=J_{e}=J_{\mathrm{CS}}$.

From eqs. (14) and (15), one can identify the following two fixed points for all $n \geq 2$ : 

(i) $\left(J_{m}^{*}, J_{e}^{*}\right)=(1,1)$,
(ii) $\left(J_{m}^{*}, J_{e}^{*}\right)=(1,0)$.

Clearly, the fixed point (i) has the $\mathrm{SU}(2 n)$ symmetry and its local Fermi-liquid property has been studied well. ${ }^{23)}$ The finite value of the coupling constant at the fixed point is an artifact of the third-order scaling and should be replaced by infinity in the exact scaling.

The trajectory of the scaling equations is shown in Fig. 1 for the case of $j=5 / 2(n=3)$. Note that $J_{m}$ is driven to strong coupling no matter how small is $J_{e}$. On the other hand, $J_{e}$ can retain the zero initial value even if $J_{m}$ is finite. This aspect is related to the particle-hole symmetry in the system as will be discussed shortly.

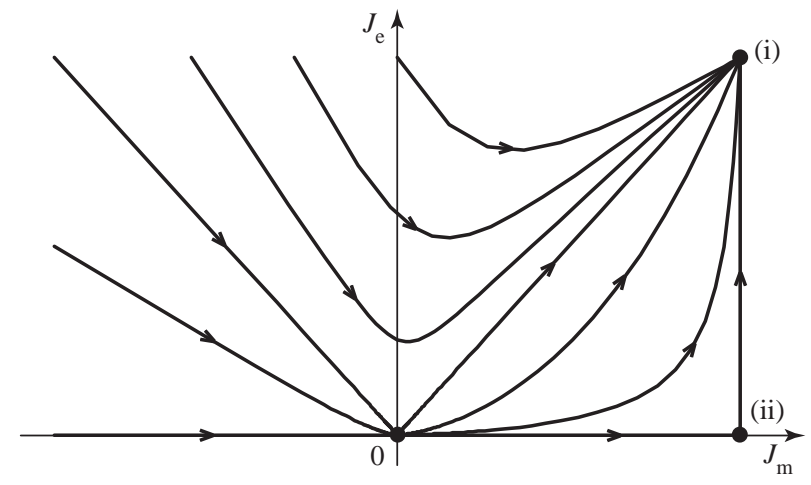

Fig. 1. The trajectory of the scaling equations for $j=5 / 2$ (3fold orbital degeneracy). There are two fixed points $\left(J_{m}^{*}, J_{e}^{*}\right)$ : (i) $(1,1)$, and (ii) $(1,0)$.

For the $\mathrm{SU}(2 n)$ CS model with $2 n=2 j+$ 1 , the characteristic energy is given by $T_{\mathrm{K}}^{\mathrm{CS}}=$ $J_{\mathrm{CS}}^{1 /(2 n)} \exp \left[-1 /\left(2 n J_{\mathrm{CS}}\right)\right]$. In the absence of $J_{e}$, on the other hand, the alternative energy scale appears: $T_{\mathrm{K}}^{m}=$ $J_{m}^{1 /(n+1)} \exp \left[-1 /(n+1) J_{m}\right]$. Figure 2 shows the solution of the third-order scaling equation for $j=5 / 2(n=3)$. Here the initial coupling constant is fixed at $J_{m 0}=0.05$ and the ratio $\alpha=J_{e} / J_{m}$ is varied. It is emphasized that $J_{e}$ remains small over a substantial energy range although $J_{m}$ is already in the strong-coupling regime. Hence one can identify two different characteristic energies in the case of $\alpha \ll 1$. The two characteristic energies merge into a single Kondo energy as $\alpha$ approaches unity.

Now we turn to discussing the property of the new fixed point (ii) with $J_{e}^{*}=0$. If one performs the particlehole transformation $c_{k m}^{\dagger} \rightarrow(-1)^{j-m} h_{-k-m}$, the coupling constants $\bar{J}_{p}$ for the transformed system are related to the original ones as

$$
\bar{J}_{p}=(-1)^{p+1} J_{p}
$$

which results from the anticommutation of fermion operators and the parity of spherical tensors. Thus the particle-hole symmetry is realized only for $J_{p}=0$ with $p$ even. The $\mathrm{SU}(2 n)$ CS model with $n>1$ breaks this symmetry since the model necessarily has $J_{e} \neq 0$.

Let us discuss how the charge Kondo effect arises, assuming that the initial coupling constants are already in the strong-coupling regime. The localized spin and one-particle or hole excitation from the Fermi sea form

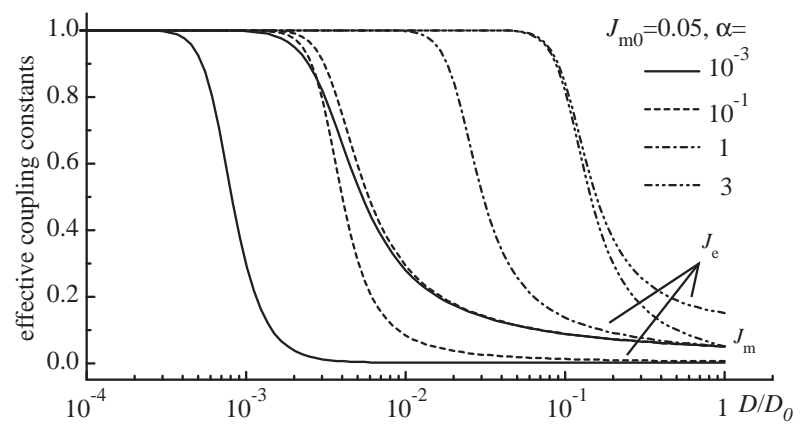

Fig. 2. The renormalization evolution for $j=5 / 2$. The initial coupling constants are fixed at $J_{m 0}=0.05$ with various values of the ratio $\alpha=J_{e 0} / J_{m 0}$.

the singlet bound state. We represent this state as $(+, 0)$ where + means the binding with a particle excitation and 0 the singlet. There is the particle-hole conjugate state $(-, 0)$ where a hole is bound to the localized spin. The energies of the two states are different by the amount $J_{e}$. If one reduces the magnitude of $J_{e}$ to zero with $J_{m}$ fixed in the strong-coupling regime, the ground state with the two-fold degeneracy in the charge degrees of freedom, $( \pm, 0)$, is realized. Therefore, at the fixed point (ii) the spin degrees of freedom is completely compensated, but the charge degrees of freedom (isospin) still remain.

We now construct the effective interaction around the fixed point (ii). In the lowest order of $t^{2} / J_{m}$, with $t$ being the hopping of innermost conduction electrons, there appear interaction terms which connect the twofold degenerate ground states. These terms accompany $c_{k m}^{\dagger} h_{-k^{\prime} m}$, or equivalently $(-1)^{j-m} c_{k m}^{\dagger} c_{k^{\prime}-m}^{\dagger}$, and the matrix element $\left\langle+, 0\left|c_{k m}^{\dagger} c_{k^{\prime}-m}^{\dagger}\right|-, 0\right\rangle$ is finite. Since the singlet state is isotropic, the pair creation with any of $m$ has the same magnitude in the interaction. Thus the effective interaction around the fixed point (ii) is given by

$$
H_{\text {eff }}^{*}=F \sum_{m=1}^{n} \vec{I}_{m} \cdot \overrightarrow{\mathcal{I}}-h \mathcal{I}_{z},
$$

where the "isospin" operators for conduction electrons are defined as

$$
\begin{aligned}
& I_{m}^{z}=\frac{1}{2}\left[\sum_{k}\left(c_{k m}^{\dagger} c_{k m}+c_{k-m}^{\dagger} c_{k-m}\right)-1\right], \\
& I_{m}^{+}=(-1)^{j-m} \sum_{k k^{\prime}} c_{k m}^{\dagger} c_{k^{\prime}-m}^{\dagger}, \quad I_{m}^{-}=\left[I_{m}^{+}\right]^{\dagger},
\end{aligned}
$$

and for localized electrons as

$$
\begin{aligned}
& \mathcal{I}_{z}=\frac{1}{2}[|+, 0\rangle\langle+, 0|-|-, 0\rangle\langle-, 0|], \\
& \mathcal{I}_{+}=|+, 0\rangle\langle-, 0|, \quad \mathcal{I}_{-}=\left[\mathcal{I}_{+}\right]^{\dagger} .
\end{aligned}
$$

The effective interaction is equivalent to that of the multi-channel Kondo model with the overscreening. The multiple channels come from the orbital degeneracy. Here we have included the weak particle-hole asymmetry 
as the second term with $h$ in eq.(23), which is of the order of $J_{e}$. Just like the multi-channel Kondo model which is unstable in the presence of magnetic field, ${ }^{22)}$ the electric coupling $J_{e}$ is the relevant perturbation in the present model.

Since the ground state has a degeneracy with $J_{e}=0$, the system exhibits an anomalous behavior with the fractional entropy as in the conventional multi-channel Kondo effect. ${ }^{20}$ ) The effective Hamiltonian is meaningful only in the case where the renormalized coupling $J_{m}$ becomes strong while $J_{e}$ still is in the weak-coupling regime. In such a case only the electric response $\chi_{p}(\omega)=$ $\left\langle T_{q}^{(p)}(\omega) T_{q}^{(p)}\right\rangle$ with even $p$ shows non-Fermi-liquid behavior. On the contrary $\chi_{p}(\omega)$ with odd $p$ shows a Fermi-liquid behavior because the spin degrees of freedom is quenched. We are now exploring quantitatively the unique behavior of the system by using the Wilson's numerical renormalization-group (NRG) method, and some anomalous properties, such as the fractional entropy, have been confirmed. ${ }^{24)}$

In summary, we have investigated a multipolar exchange model with the rotational symmetry. Respecting the different time-reversal properties of operators, we find a new fixed point where a non-Fermi liquid appears as a result of the Kondo effect in the charge sector. Although the fixed point is unstable against any perturbation breaking the particle-hole symmetry, the non-Fermi-liquid behavior may be observed over a substantial energy range in the case of $J_{e} \ll J_{m}$. Thus, more quantitative investigation of the model is highly desired.

\section{Acknowledgements}

This work was supported by Grand-in-Aid for encouragement of Young Scientists from the Ministry of Education, Science and Culture of Japan and also by CREST from the Japan Science and Technology Corporation.

1) M. Hiroi, S. Kobayashi, M. Sera, N. Kobayashi, and S. Kunii: Phys. Rev. Lett. 81 (1998) 2510, and references therein.

2) S. Nakamura, O. Suzuki, T. Goto, S. Sakatsume, T. Matsumura, and S. Kunii: J. Phys. Soc. Jpn. 66 (1997) 552.

3) T. Tayama, T. Sakakibara, K. Tenya, H. Amitsuka, and S. Kunii: J. Phys. Soc. Jpn. 66 (1997) 2268.

4) A. P. Ramirez: J. Phys. C9 (1997) 8171, and references therein.

5) Y. Murakami, H. Kawada, H. Kawata, M. Tanaka, T. Arima, Y. Moritomo, and Y. Tokura: Phys. Rev. Lett. 80 (1998) 1932.

6) K. I. Kugel and D. I. Khomskii: Sov. Phys. JETP 37 (1973) 725 .

7) D. Schmitt and P. M. Levy: J. Magn. \& Magn. Mater. 49 (1985) 15.

8) R. Shiina, H. Shiba and P. Thalmeier: J. Phys. Soc. Jpn. 66 (1997) 1741, and references therein.

9) N. Fukushima and Y. Kuramoto: J. Phys. Soc. Jpn. 67 (1998) 2460.

10) B. Sutherland: Phys. Rev. B12 (1975) 3795.

11) I. Affleck: Nucl. Phys. B265 (1986) 409.

12) Y. Yamashita, N. Shibata and K. Ueda: Phys. Rev. B58 (1998) 9114.

13) Y. Q. Li, M. Ma, D. N. Shi and F. C. Zhang: Phys. Rev. Lett. 81 (1998) 3527.

14) B. Frischmuth, F. Mila and M. Troyer: Phys. Rev. Lett. 82 (1999) 835.
15) L. L. Hirst: Z. Phys. 244 (1971) 230; Adv. Phys. 27 (1978) 231.

16) E. Pavarini and L. C. Andreani: Phys. Rev. B56 (1997) 5073.

17) H. Kusunose and Y. Kuramoto: Phys. Rev. B59 (1999) 1902.

18) L. D. Landau and E. M. Lifshitz: Quantum Mechanics (Pergamon Press, London, 1958).

19) In leading to the usual multi-channel Kondo exchange interaction, the coupling with different $j$ partial waves is essential.

20) D. L. Cox and A. Zawadowski: Adv. Phys. 47 (1998) 599.

21) Y. Kuramoto: Eur. Phys. J. B5 (1998) 457.

22) H. Pang and D. L. Cox: Phys. Rev. B44 (1991) 9454.

23) V. T. Rajan: Phys. Rev. Lett. 51 (1983) 308.

24) H. Kusunose and Y. Kuramoto: in preparation. 